\title{
Association of dietary fat, vegetables and antioxidant micronutrients with skin ageing in Japanese women
}

\author{
Chisato Nagata ${ }^{1}$, Kozue Nakamura ${ }^{1}$, Keiko Wada ${ }^{1}$, Shino Oba ${ }^{1}$, Makoto Hayashi $^{2}$, Noriyuki Takeda ${ }^{3}$ \\ and Keigo Yasuda ${ }^{2}$ \\ ${ }^{1}$ Department of Epidemiology \& Preventive Medicine, Gifu University Graduate School of Medicine, Gifu 501-1194, Japan \\ ${ }^{2}$ Department of Internal Medicine, Matsunami General Hospital, Gifu 501-6061, Japan \\ ${ }^{3}$ Department of Endocrinology and Metabolism, Murakami Memorial Hospital, Asahi University, Gifu 500-8523, Japan
}

(Received 25 August 2009 - Revised 9 November 2009 - Accepted 16 November 2009 - First published online 20 January 2010 )

Daily diet may have implications for skin ageing. However, data on the relationship between diet and the parameters of skin conditions are scarce. The present study aimed to examine the associations of biophysical properties of the skin of women with intakes of fats and antioxidant micronutrients as well as food groups as sources of these nutrients. In a cross-sectional study, we measured the hydration, surface lipids and elasticity of the skin of 716 Japanese women using non-invasive techniques. The extent of facial wrinkles in the crow's-foot area was determined by observation using the Daniell scale. Each subject's usual diet was determined with the use of a validated FFQ. After controlling for covariates including age, smoking status, BMI and lifetime sun exposure, the results showed that higher intakes of total fat, saturated fat and monounsaturated fat were significantly associated with increased skin elasticity. A higher intake of green and yellow vegetables was significantly associated with a decreased Daniell wrinkling score. Intake of saturated fat was significantly inversely associated with the Daniell wrinkling score after additional adjustment for green and yellow vegetable intake. Further studies with more accurate measurement methods are needed to investigate the role of daily diet in skin ageing.

Dietary fats: Skin elasticity: Facial wrinkling: Vegetables

Interest in the impact of ageing on the function and appearance of the skin has been increasing ${ }^{(1)}$. Aged skin has several typical characteristics, including fine wrinkles, dryness, sallowness and loss of elasticity ${ }^{(2)}$. These characteristics are thought to result from a complex process controlled by both environmental and genetic factors ${ }^{(3)}$. Among environmental factors, sun exposure and cigarette smoking have long been investigated as risk factors for premature skin ageing ${ }^{(4-6)}$. Large amounts of micronutrients such as those with antioxidant capacity are present in the skin and are suggested to contribute to the maintenance of skin health ${ }^{(7)} \cdot n-3$ PUFA, especially EPA and DHA, are known to have anti-inflammatory effects and thus are thought to have a protective effect against inflammatory skin disorders ${ }^{(8)}$. The effects of the antioxidant vitamins and carotenoids or fish oil on conditions of the skin have been examined in some intervention studies ${ }^{(9)}$. However, these studies are limited by the use of supplements. Little is known about the effects of the long-term consumption of vitamins, carotenoids, or fatty acids at nutritional amounts in the daily diet.

To our knowledge, only three studies have examined associations between daily diet and skin conditions ${ }^{(10-12)}$. Purba et al. ${ }^{(10)}$ assessed actinic skin wrinkling of the back of the hand based on the grading of cutaneous microtopographs of 453 men and women living in Europe and Australia. Using a non-invasive technique, Boelsma et al. ${ }^{(11)}$ measured the hydration and surface $\mathrm{pH}$ of the skin of the right arm and the sebum content of the forehead of 302 Dutch men and women. Cosgrove $e t$ al. ${ }^{(12)}$ assessed the appearance of wrinkles, senile dryness and skin atrophy, which were classified as present or absent based on observations by dermatologists, among 4025 American women. These studies revealed that certain types of fats or antioxidant micronutrients were associated with some measured skin properties. However, considering that the sites of the skin measured, the parameters of skin conditions, and the skin assessment methods varied among these studies, cumulated data are still scarce and far from conclusive. In the present study of Japanese women, we examined cross-sectional relationships between dietary intakes of fats and antioxidant micronutrients as well as food groups as sources of these nutrients and biophysical properties of the skin, including hydration, sebum content, elasticity and wrinkle appearance.

\section{Materials and methods}

\section{Study population}

The present study was part of a larger study designed to assess the relationships among lifestyle, environmental factors and 
women's health ${ }^{(13)}$. Study subjects were participants in a medical health check-up programme provided by a general hospital in Gifu, Japan, between October 2003 and March 2006. A total of 2073 individuals, including return visitors to the programme during the study period, were invited to join the study, and 1545 agreed to participate (response proportion: $74.5 \%$ ). When the response proportion was calculated for only new visitors to the programme during the study period, it was $83.2 \%$ (1103 out of 1325 individuals). The details are described elsewhere ${ }^{(13)}$. The present study was conducted according to the guidelines laid down in the Declaration of Helsinki and all procedures involving human subjects were approved by the ethical board of the Gifu University Graduate School of Medicine. Written informed consent was obtained from all subjects.

\section{Dietary measurement}

The participants responded to a self-administered questionnaire that included questions on demographic characteristics, smoking and drinking habits, diet, exercise, sun exposure, and medical and reproductive histories. Intakes of foods and nutrients were estimated based on a validated 169-item semi-quantitative FFQ using the Japanese Standard Tables of Food Composition, 4th and 5th editions, and fatty acid composition data published by Sasaki et al. ${ }^{(14)}$. Intake of long-chain $n$-3 PUFA was calculated as the sum of EPA and DHA. Detailed information on the questionnaire, including its validity and reproducibility examined in other samples, has been described elsewhere ${ }^{(15)}$. The Spearman correlation coefficients between the questionnaire and twelve daily diet records kept over a 1-year period for major and micronutrients ranged from 0.29 for carotene $(\alpha$ - and $\beta$-carotenes plus cryptoxanthin; expressed as $\beta$-carotene equivalents) to 0.73 for $\mathrm{Ca}$. Those for intakes of total fat, saturated fats, monounsaturated fat, polyunsaturated fat, and green and yellow vegetables were greater than $0 \cdot 50$. Our questionnaire was designed to measure an individual's relative intake of foods and nutrients rather than absolute values. The means estimated from the FFQ were generally higher than those estimated from twelve daily diet records. Although we presented the mean values of dietary intake, some of them may have been overestimated by our questionnaire.

\section{Assessment of other exposure variables}

Data on lifetime sun exposure were collected through faceto-face interviews. Subjects identified periods in their lives during which they had had stable patterns of outdoor activity. For each of these periods, the subjects were asked about the amount of time they spent outdoors per $d$ during a typical week. Weekday and weekend exposures were recorded separately. We estimated the total time spent outdoors from age 12 years until the day of the interview. Current use of sunscreens during summer was also recorded. Exercise was assessed by asking the average time spent (h per week) performing various kinds of activities during the past 1 year. The details, including validity, are described elsewhere ${ }^{(16)}$.

\section{Assessment of skin ageing}

Skin properties were measured by two investigators. The subjects rested for about $15 \mathrm{~min}$ in a room for skin measurements. The cosmetics on the selected sites were removed by the investigators. All the measurements were performed in triplicate.

Skin hydration was measured with a corneometer (CM825; Courage and Khazaka, Electronic GmbH, Cologne, Germany). The probe was applied to the skin of the inner side of the right upper arm for $1 \mathrm{~s}$ at a pressure of $3.5 \mathrm{~N} / \mathrm{cm}^{2}$. The degree of epidermal humidity is indicated in system-specific units.

Skin surface lipids composed of sebum and corneal lipids were measured with a Sebumeter (SM810; Courage and Khazaka, Electronic $\mathrm{GmbH}$ ). This device, which works on the principle of photometry, has a special plastic strip that becomes transparent when it absorbs fat. The measuring head equipped with this strip was pressed on the skin of the forehead with a constant pressure of $10 \mathrm{~N} / 64 \mathrm{~mm}^{2}$ for $30 \mathrm{~s}$. The value displayed corresponded to the amount of sebum on the skin surface in $\mu \mathrm{g} / \mathrm{cm}^{2}$.

Skin elasticity of the inner side of the right upper arm was measured with a cutometer (SEM575; Courage and Khazaka, Electronic $\mathrm{GmbH}$ ). The measurements were performed with a negative pressure of $350 \mathrm{mbar}$, a suction time of $2 \mathrm{~s}$ and a relaxation phase of $2 \mathrm{~s}$. The assessment parameters consisted of the Ur/Uf coefficient, which represents the ability of the skin to regain its initial position after deformation.

The extent of facial wrinkles in the crow's-foot area was determined by the Daniell scale ${ }^{(4)}$ ranging from grade I (essentially no wrinkling) to grade VI (profound wrinkling extending over most of the face).

\section{Data analyses}

A total of 1086 women participated in the skin measurements. Women who had cancer ( $n$ 24) or collagen diseases ( $n$ 11) such as rheumatism and systemic lupus erythematosus were excluded from the present analyses. Additionally, 319 women who had not responded to the dietary questionnaire ( $n$ 259) or those with incomplete or unreliable responses to the dietary questionnaire (criteria shown elsewhere ${ }^{(17)} ; n 60$ ) and sixteen women who did not complete the interview of lifetime sun exposure were excluded from the present analyses. Thus, 716 women aged 20-74 years comprised the study population. Skin elasticity data were unavailable for 124 women as the device was out of order during their checkups. Women who did not respond to the dietary questionnaire were more likely to be older than those who did. After controlling for age, the means of skin property variables of the nonresponders were similar to those who responded to the dietary frequency questionnaire except for a lower mean value for skin surface lipids.

For statistical analysis, dietary intakes were adjusted for total energy after log-transformation by using the residual method proposed by Willett ${ }^{(18)}$. Quintiles of the dietary variables were derived based on their distribution in the total population. The means of skin properties for each quintile were provided using analysis of covariate models. A linear trend was assessed using continuous values. Since skin hydration was correlated with room temperature and humidity 
Table 1. Characteristics of study subjects and their correlations with skin parameters $(n 716)$ (Mean values and standard deviations, percentages and correlations)

\begin{tabular}{|c|c|c|c|c|c|c|}
\hline & \multicolumn{2}{|c|}{ Characteristics } & \multicolumn{4}{|c|}{ Correlations $†$} \\
\hline & Mean & SD & Hydration & Surface lipids & Elasticity & Facial wrinkles \\
\hline Age (years) & $43 \cdot 3$ & $8 \cdot 2$ & 0.04 & $-0 \cdot 20^{\star *}$ & $-0.32^{* *}$ & $0.70^{\star *}$ \\
\hline $\operatorname{BMI}\left(\mathrm{kg} / \mathrm{m}^{2}\right)$ & $21 \cdot 3$ & $2 \cdot 9$ & 0.12 & 0.02 & $0.13^{\star *}$ & 0.03 \\
\hline Education (years) & 13.7 & $2 \cdot 0$ & -0.14 & 0.005 & -0.02 & 0.02 \\
\hline Exercise (METs h/week) & $26 \cdot 2$ & $33 \cdot 0$ & -0.0003 & -0.03 & -0.06 & -0.07 \\
\hline Sun exposure (cumulative h) & 17430 & 10264 & 0.01 & 0.02 & -0.01 & $0 \cdot 17^{\star \star}$ \\
\hline Alcohol intake $(\mathrm{ml} / \mathrm{d})$ & $6 \cdot 6$ & $16 \cdot 0$ & 0.03 & 0.05 & -0.03 & 0.05 \\
\hline Not married (\%) & \multicolumn{2}{|c|}{$18 \cdot 7$} & -0.04 & 0.001 & -0.07 & -0.03 \\
\hline Postmenopausal (\%) & \multicolumn{2}{|c|}{$19 \cdot 1$} & -0.03 & -0.05 & -0.03 & 0.04 \\
\hline Current smokers (\%) & \multicolumn{2}{|c|}{$6 \cdot 0$} & -0.01 & 0.07 & -0.03 & $0 \cdot 10^{\star \star}$ \\
\hline Ex-smokers (\%) & \multicolumn{2}{|c|}{3.9} & 0.02 & -0.01 & 0.005 & 0.03 \\
\hline Current HRT use (\%) & \multicolumn{2}{|c|}{3.4} & 0.03 & 0.05 & 0.01 & -0.05 \\
\hline Current OC use (\%) & \multicolumn{2}{|c|}{0.8} & 0.01 & 0.002 & 0.005 & -0.03 \\
\hline
\end{tabular}

(Spearman's $r$ were -0.19 and 0.62 , respectively), the results under restricted ranges (temperature, $20-25^{\circ} \mathrm{C}$; humidity, $40-60 \% ; n$ 209) are shown. Room temperature and humidity were dealt with as covariates because they were strongly correlated with skin hydration ( $r 0.09$ for temperature and $r 0.50$ for humidity). Although the correlation coefficients of room temperature and humidity with other skin variables were less than 0.08, they were also included as covariates. The known or suspected risk factors for skin conditions, such as age, BMI, smoking status and sunlight exposure ${ }^{(19)}$, were included in the models as covariates in addition to room temperature and humidity. The indicator for raters was also included as a covariate. All the statistical analyses were performed using SAS programs (SAS Institute Inc., Cary, NC, USA). Significance was defined as two-sided $P<0.05$.

\section{Results}

Table 1 shows the characteristics of the study subjects and their correlations with skin parameters. Age, BMI, smoking status and sunlight exposure were significantly correlated with one or more parameters. Table 2 shows the intercorrelations among the skin parameters.

Table 3 shows the means for each skin parameter according to the quintile of the selected nutrient or food intake after controlling for the covariates. Surface lipids were significantly positively associated with fresh and processed meats. Skin elasticity was significantly positively associated with total fat, saturated fat and monounsaturated fat, although the differences in the mean elasticity between the highest and the lowest quintiles of these types of fats were less than $2 \%$. The Daniell wrinkling score was significantly inversely associated with green and yellow vegetable intake. Saturated fat was marginally significantly associated with Daniell's wrinkling score, but this association obtained significance after additional adjustment for green and yellow vegetable intake $(P=0.049)$. The association between the Daniell wrinkling score and green and yellow vegetable intake remained statistically significant after additional adjustment for saturated fat intake $(P=0 \cdot 04)$. Vitamins $\mathrm{C}$ and $\mathrm{E}$ and $\mathrm{Zn}$ were unrelated to any parameter of the skin measurements.

\section{Discussion}

Skin elasticity on the forearm has been reported to decrease with chronological age ${ }^{(20,21)}$. In the present study, skin elasticity was significantly inversely correlated with facial wrinkle score, suggesting the importance of this parameter in skin ageing. We observed that higher intakes of total fat, saturated fat and monounsaturated fat were moderately but significantly associated with increased elasticity. A higher intake of longchain $n-3$ fatty acids was marginally significantly associated with increased elasticity. The associations with elasticity did not differ greatly by type of fat, although polyunsaturated fat was not significantly associated with skin elasticity. Fats provide building blocks for many components of epidermal and dermal tissues, and they are sources of energy in cell proliferation, maturation and homeostasis ${ }^{(22)}$. Fats are sensitive to the oxidation process ${ }^{(22)}$. However, maintenance of collagen and elastic fibres may require adequate amount of fat. Higher saturated fat intake was also significantly associated with a decreased facial wrinkling, suggesting a favourable effect of fat. None of the previous cross-sectional studies on diet and skin conditions ${ }^{(10-12)}$ included the measurement of skin elasticity but addressed the association of fat intake with other parameters of skin conditions. Purba et al. ${ }^{(10)}$ found that higher intakes of total fat and monounsaturated fat were significantly associated with decreased wrinkling on

Table 2. Intercorrelations among skin parameters (Spearman correlation coefficients)

\begin{tabular}{|c|c|c|c|c|}
\hline & Hydration & Surface lipids & Elasticity & Facial wrinkles \\
\hline Hydration & 1.00 & 0.02 & 0.03 & 0.12 \\
\hline Surface lipids & - & 1.00 & $0.13^{\star *}$ & $-0.11^{\star *}$ \\
\hline Elasticity & - & - & 1.00 & $-0 \cdot 30^{\star \star}$ \\
\hline Facial wrinkles & - & - & - & 1.00 \\
\hline
\end{tabular}

${ }^{* *} P<0.01$ 
Table 3. Adjusted* means of skin parameters according to quintiles (Q) of selected dietary variables

\begin{tabular}{|c|c|c|c|c|c|}
\hline & Median† & Hydration (arbitrary units) & Surface lipids $\left(\mu \mathrm{g} / \mathrm{cm}^{2}\right)$ & Elasticity (\%) & Facial wrinkles (Daniell score) \\
\hline \multicolumn{6}{|l|}{ Total fat $(\mathrm{g} / \mathrm{d})$} \\
\hline Q1 & $50 \cdot 8$ & 41.4 & $68 \cdot 6$ & $81 \cdot 8$ & 2.48 \\
\hline Q2 & $57 \cdot 3$ & $41 \cdot 6$ & $69 \cdot 8$ & $82 \cdot 4$ & 2.48 \\
\hline Q3 & $62 \cdot 4$ & $40 \cdot 2$ & $75 \cdot 9$ & $83 \cdot 1$ & $2 \cdot 57$ \\
\hline Q4 & $67 \cdot 2$ & $40 \cdot 2$ & $73 \cdot 3$ & $82 \cdot 8$ & $2 \cdot 41$ \\
\hline Q5 & 73.8 & $40 \cdot 0$ & $72 \cdot 3$ & $83 \cdot 6$ & $2 \cdot 38$ \\
\hline$P$ for trend & & 0.28 & 0.26 & 0.007 & 0.17 \\
\hline \multicolumn{6}{|c|}{ Saturated fat $(\mathrm{g} / \mathrm{d})$} \\
\hline Q1 & $13 \cdot 6$ & $41 \cdot 3$ & $68 \cdot 2$ & $82 \cdot 0$ & $2 \cdot 51$ \\
\hline Q2 & $16 \cdot 2$ & 41.5 & $69 \cdot 5$ & $82 \cdot 8$ & 2.54 \\
\hline Q3 & $18 \cdot 2$ & $39 \cdot 3$ & $72 \cdot 8$ & $82 \cdot 7$ & 2.43 \\
\hline Q4 & $19 \cdot 8$ & 41.5 & $77 \cdot 0$ & 82.9 & 2.45 \\
\hline Q5 & 23.4 & $40 \cdot 4$ & $72 \cdot 3$ & $83 \cdot 3$ & $2 \cdot 39$ \\
\hline$P$ for trend & & 0.64 & 0.12 & 0.03 & 0.06 \\
\hline \multicolumn{6}{|c|}{ Monounsaturated fat $(\mathrm{g} / \mathrm{d})$} \\
\hline Q1 & $16 \cdot 3$ & 41.3 & $68 \cdot 2$ & $81 \cdot 7$ & $2 \cdot 54$ \\
\hline Q2 & $19 \cdot 2$ & $42 \cdot 0$ & $70 \cdot 9$ & $82 \cdot 2$ & 2.46 \\
\hline Q3 & $21 \cdot 2$ & 40.5 & $72 \cdot 8$ & 83.2 & 2.44 \\
\hline Q4 & $23 \cdot 2$ & 39.4 & 74.4 & $83 \cdot 0$ & 2.52 \\
\hline Q5 & $26 \cdot 1$ & $40 \cdot 0$ & 73.5 & $83 \cdot 6$ & $2 \cdot 36$ \\
\hline$P$ for trend & & 0.18 & 0.07 & 0.007 & 0.34 \\
\hline \multicolumn{6}{|c|}{ Polyunsaturated fat $(\mathrm{g} / \mathrm{d})$} \\
\hline Q1 & $12 \cdot 1$ & $42 \cdot 8$ & 74.5 & $82 \cdot 5$ & 2.43 \\
\hline Q2 & $14 \cdot 0$ & $39 \cdot 8$ & $72 \cdot 0$ & $82 \cdot 3$ & 2.51 \\
\hline Q3 & $15 \cdot 3$ & $40 \cdot 3$ & $70 \cdot 1$ & $82 \cdot 6$ & 2.44 \\
\hline Q4 & $16 \cdot 6$ & $40 \cdot 7$ & $70 \cdot 9$ & 83.0 & 2.45 \\
\hline Q5 & 18.9 & 39.8 & $72 \cdot 3$ & 83.2 & $2 \cdot 48$ \\
\hline$P$ for trend & & 0.27 & 0.72 & 0.19 & $0 \cdot 11$ \\
\hline \multicolumn{6}{|c|}{ Long-chain $n-3$ fatty acids $(\mathrm{mg} / \mathrm{d})$} \\
\hline Q1 & 366 & $42 \cdot 0$ & $70 \cdot 1$ & 81.9 & 2.46 \\
\hline Q2 & 508 & 41.4 & $71 \cdot 2$ & $82 \cdot 6$ & $2 \cdot 49$ \\
\hline Q3 & 630 & $40 \cdot 1$ & 79.0 & 83.5 & $2 \cdot 44$ \\
\hline Q4 & 817 & $39 \cdot 2$ & $70 \cdot 7$ & $82 \cdot 7$ & $2 \cdot 52$ \\
\hline Q5 & 1121 & $40 \cdot 7$ & $68 \cdot 7$ & 83.0 & 2.41 \\
\hline$P$ for trend & & 0.21 & 0.87 & 0.09 & 0.95 \\
\hline \multicolumn{6}{|c|}{ Carotene $(\mathrm{mg} / \mathrm{d})$} \\
\hline Q1 & 2973 & 41.6 & 69.7 & $82 \cdot 6$ & 2.54 \\
\hline Q2 & 4014 & $40 \cdot 0$ & $77 \cdot 3$ & 83.4 & 2.57 \\
\hline Q3 & 4848 & 39.7 & 67.4 & 82.5 & 2.45 \\
\hline Q4 & 6188 & $40 \cdot 6$ & 73.9 & 82.6 & $2 \cdot 39$ \\
\hline Q5 & 8820 & $41 \cdot 2$ & 71.5 & $82 \cdot 6$ & $2 \cdot 36$ \\
\hline$P$ for trend & & 0.69 & 0.53 & 0.56 & 0.08 \\
\hline \multicolumn{6}{|c|}{ Vitamin $C(\mathrm{mg} / \mathrm{d})$} \\
\hline Q1 & 90.9 & 41.4 & 73.4 & $82 \cdot 9$ & 2.48 \\
\hline Q2 & $115 \cdot 6$ & 38.7 & 63.7 & 82.5 & 2.43 \\
\hline Q3 & $141 \cdot 8$ & $41 \cdot 8$ & 74.8 & $82 \cdot 3$ & 2.51 \\
\hline Q4 & $170 \cdot 2$ & $40 \cdot 3$ & $72 \cdot 8$ & $82 \cdot 7$ & 2.35 \\
\hline Q5 & $235 \cdot 3$ & 41.0 & $75 \cdot 1$ & $83 \cdot 1$ & 2.55 \\
\hline$P$ for trend & & 0.95 & 0.47 & 0.76 & 0.78 \\
\hline \multicolumn{6}{|c|}{ Vitamin $E(\mathrm{mg} / \mathrm{d})$} \\
\hline Q1 & 8.4 & $40 \cdot 6$ & 74.8 & $82 \cdot 3$ & 2.51 \\
\hline Q2 & $9 \cdot 6$ & 41.2 & 70.9 & $82 \cdot 8$ & 2.54 \\
\hline Q3 & $10 \cdot 4$ & $40 \cdot 3$ & 73.9 & $82 \cdot 9$ & $2 \cdot 52$ \\
\hline Q4 & 11.5 & $40 \cdot 0$ & $70 \cdot 4$ & $82 \cdot 8$ & $2 \cdot 30$ \\
\hline Q5 & $13 \cdot 2$ & 41.3 & 69.8 & 82.9 & 2.45 \\
\hline$P$ for trend & & 0.88 & 0.54 & 0.49 & 0.27 \\
\hline \multicolumn{6}{|l|}{$\mathrm{Zn}(\mathrm{mg} / \mathrm{d})$} \\
\hline Q1 & $9 \cdot 4$ & $41 \cdot 2$ & $69 \cdot 1$ & 82.5 & 2.56 \\
\hline Q2 & $10 \cdot 2$ & 39.0 & $72 \cdot 1$ & $82 \cdot 9$ & $2 \cdot 47$ \\
\hline Q3 & $10 \cdot 9$ & $40 \cdot 9$ & 71.5 & 82.5 & $2 \cdot 41$ \\
\hline Q4 & 11.5 & 41.5 & $72 \cdot 0$ & 82.5 & 2.47 \\
\hline Q5 & $12 \cdot 6$ & 40.4 & $75 \cdot 2$ & 83.3 & $2 \cdot 40$ \\
\hline$P$ for trend & & 0.53 & 0.36 & 0.18 & 0.36 \\
\hline \multicolumn{6}{|c|}{ Fresh and processed meats $(\mathrm{g} / \mathrm{d})$} \\
\hline Q1 & 37.9 & $40 \cdot 9$ & 64.0 & $82 \cdot 6$ & $2 \cdot 38$ \\
\hline Q2 & 58.3 & $42 \cdot 1$ & $72 \cdot 1$ & $82 \cdot 6$ & 2.46 \\
\hline Q3 & $72 \cdot 4$ & 41.9 & $71 \cdot 2$ & $82 \cdot 4$ & 2.50 \\
\hline Q4 & $90 \cdot 6$ & $38 \cdot 3$ & $73 \cdot 2$ & $82 \cdot 8$ & 2.59 \\
\hline Q4 & $123 \cdot 0$ & 39.5 & 79.5 & 83.3 & $2 \cdot 39$ \\
\hline$P$ for trend & & 0.25 & 0.01 & 0.37 & 0.65 \\
\hline
\end{tabular}


Table 3. Continued

\begin{tabular}{|c|c|c|c|c|c|}
\hline & Median† & Hydration (arbitrary units) & Surface lipids $\left(\mu \mathrm{g} / \mathrm{cm}^{2}\right)$ & Elasticity (\%) & Facial wrinkles (Daniell score) \\
\hline \multicolumn{6}{|c|}{ Fish and shell fish $(\mathrm{g} / \mathrm{d})$} \\
\hline Q1 & 40.5 & 41.9 & $77 \cdot 6$ & $82 \cdot 9$ & 2.35 \\
\hline Q2 & $58 \cdot 0$ & $38 \cdot 8$ & $67 \cdot 0$ & $82 \cdot 0$ & $2 \cdot 46$ \\
\hline Q3 & 71.4 & $40 \cdot 9$ & $74 \cdot 2$ & 83.2 & 2.56 \\
\hline Q4 & $86 \cdot 0$ & $40 \cdot 8$ & $70 \cdot 2$ & $82 \cdot 8$ & 2.50 \\
\hline Q5 & 114.4 & 41.0 & $70 \cdot 6$ & $82 \cdot 7$ & 2.46 \\
\hline$P$ for trend & & 0.29 & 0.37 & 0.56 & 0.42 \\
\hline \multicolumn{6}{|c|}{ Green and yellow vegetables $(\mathrm{g} / \mathrm{d})$} \\
\hline Q1 & 62.9 & $40 \cdot 3$ & 71.6 & 82.5 & 2.51 \\
\hline Q2 & $91 \cdot 7$ & 41.4 & $75 \cdot 9$ & $83 \cdot 6$ & 2.58 \\
\hline Q3 & $120 \cdot 1$ & $42 \cdot 1$ & $66 \cdot 7$ & 82.4 & 2.46 \\
\hline Q4 & $156 \cdot 9$ & 38.2 & $70 \cdot 1$ & $82 \cdot 9$ & 2.40 \\
\hline Q5 & $250 \cdot 2$ & 41.6 & 75.5 & $82 \cdot 3$ & 2.37 \\
\hline$P$ for trend & & 0.82 & 0.36 & 0.91 & 0.04 \\
\hline \multicolumn{6}{|c|}{ Other vegetables $(\mathrm{g} / \mathrm{d})$} \\
\hline Q1 & 121.4 & $41 \cdot 1$ & $72 \cdot 1$ & $82 \cdot 8$ & 2.48 \\
\hline Q2 & 179.5 & $39 \cdot 1$ & $79 \cdot 8$ & $83 \cdot 2$ & 2.49 \\
\hline Q3 & $206 \cdot 3$ & 41.2 & 70.6 & $82 \cdot 6$ & 2.41 \\
\hline Q4 & $247 \cdot 1$ & $40 \cdot 7$ & $66 \cdot 8$ & $82 \cdot 4$ & $2 \cdot 41$ \\
\hline Q5 & 335.6 & 41.4 & 70.5 & $82 \cdot 7$ & 2.52 \\
\hline$P$ for trend & & 0.45 & 0.57 & 0.32 & 0.73 \\
\hline \multicolumn{6}{|l|}{ Fruits $(g / d)$} \\
\hline Q1 & 34.6 & $40 \cdot 2$ & $77 \cdot 6$ & 82.5 & $2 \cdot 56$ \\
\hline Q2 & $61 \cdot 3$ & 39.8 & $66 \cdot 3$ & 83.0 & $2 \cdot 43$ \\
\hline Q3 & 89.4 & $40 \cdot 8$ & 68.9 & $82 \cdot 1$ & $2 \cdot 42$ \\
\hline Q4 & $140 \cdot 3$ & 41.6 & 71.9 & $82 \cdot 8$ & 2.47 \\
\hline Q5 & 229.0 & $40 \cdot 9$ & $75 \cdot 0$ & 83.3 & 2.43 \\
\hline$P$ for trend & & 0.55 & 0.69 & 0.12 & 0.24 \\
\hline
\end{tabular}

*Adjusted for total energy, age, BMI, smoking status, cumulative sun exposure, rater, and room temperature and humidity.

$\dagger$ Adjusted for total energy.

the back of the hand; this result does not contradict the present results. However, a higher intake of butter or margarine was associated with increased wrinkling. Boelsma et al. ${ }^{(11)}$ observed that higher intakes of total fat, saturated fat and monounsaturated fat were significantly associated with decreased skin hydration of the skin on the right arm. In the study reported by Cosgrave et al. ${ }^{(12)}$, a higher intake of fat was associated with wrinkled appearance and senile dryness, but a higher intake of linoleic acid was associated with decreased senile dryness. The results of these studies suggested an unfavourable role of certain types of fat in skin health. Fat consumption in the present study subjects, like that in other Japanese populations, was low as compared with those among Western populations, which may partially explain the discrepancies in the results.

Skin elasticity has been reported to be improved by hormone replacement therapy ${ }^{(23,24)}$. Higher fat intake has been associated with increased endogenous oestrogen concentrations of women in some studies ${ }^{(25,26)}$. The observed association of fat intake with skin elasticity may be explained by oestrogen profile. We expected that alcohol and phytooestrogens such as soya isoflavones might mimic the effects of oestrogen on the skin. However, neither alcohol nor dietary soya was associated with skin elasticity or the other parameters (data not shown).

Dietary supplementation with antioxidant vitamins or carotenoids has shown photoprotective effects on the skin in some studies ${ }^{(27-29)}$. In the present study, a higher intake of green and yellow vegetables was associated with decreased facial wrinkling. Carotene, which is abundant in green and yellow vegetables, was marginally inversely associated with facial wrinkling. However, we cannot deny a possibility that the observed inverse association of green and yellow vegetable intake with facial wrinkling may be due to certain nutrients other than carotene. Purba et al. ${ }^{(10)}$ also observed that a higher intake of vegetables was associated with less actinic damage of the back of the hand.

The FFQ, like all methods of dietary assessment, is subject to measurement error. In addition, the measurements of skin properties are likely to be affected by environmental conditions, such as the temperature and humidity of the room for the measurements. A room humidity of between 40 and $60 \%$ is generally recommended for measurements of skin hydration ${ }^{(30)}$. However, we noticed that the room humidity affected the skin hydration, even in such a restricted range. Nonetheless, the findings were not altered in subanalyses with narrow ranges for room temperature and humidity. It is unlikely that the measurement errors in the skin parameters are dependent on diet. Thus, the observed associations were modest but likely to be underestimated. The homogeneity of diet among the study subjects might have precluded us detecting a significant association.

Because of the cross-sectional nature of the data, no causal inferences can be drawn regarding any of the associations observed. The relatively narrow age range and the inclusion of women only are also limitations. Although fats and green and yellow vegetables were associated with some skin parameters of Japanese women, these modest associations may be by chance due to multiple testing. Given the small number of epidemiological studies on diet and skin health 
and the potential for these studies to promote a healthy diet, further studies with more accurate measurement methods are needed.

\section{Acknowledgements}

The present study was supported in part by grants from the Ministry of Education, Culture, Sports, Science, and Technology, Japan.

C. N. initiated and organised the study, and wrote the manuscript. K. N. and K. W. were involved in the analyses and interpretation of the study. S. O. helped to design the analytic strategy. M. H., N. T. and K. Y. helped to supervise the field activities and interpret the data.

None of the authors had a conflict of interest in relation to the present study.

\section{References}

1. Field KA (2000) Skin breakthroughs in the year 2000. Int $J$ Fertil Womens Med 45, 175-181.

2. Makrantonaki E \& Zouboulis CC (2007) Characteristics and pathomechanisms of endogenously aged skin. Dermatology 214, 352-360.

3. Shekar SN, Luciano M, Duffy DL, et al. (2005) Genetic and environmental influences on skin pattern deterioration. J Invest Dermatol 125, 1119-1129.

4. Daniell HW (1971) Smoker's wrinkles. Ann Intern Med 75, 873-880.

5. Ernster VL, Grady D, Miike R, et al. (1995) Facial wrinkling in men and women by smoking status. Am J Public Health 85, $78-82$.

6. Richard S, de Rigai J, de Lacharriere O, et al. (1994) Noninvasive measurement of the effect of lifetime exposure to the sun on the aged skin. Photodermatol Photoimmunol Photomed 10, 164-169.

7. Keller KL \& Fenske NA (1998) Use of vitamins A, C, and E and related conpounds in dermatology: a review. J Am Acad Dermatol 39, 611-625.

8. Storey A, McArdle F, Friedmann PS, et al. (2005) Eicosapentaenoic acid and docosahexaenoic acid reduce UVB- and TNF- $\alpha$-induced IL-8 secretion in keratinocytes and UVBinduced IL-8 in fibroblasts. J Invest Dermatol 124, 248-255.

9. Boelsma E, Hendriks HFJ \& Roza L (2001) Nutritional skin care: health effects of micronutrients and fatty acids. $A m J$ Clin Nutr 73, 853-864

10. Purba MB, Kouria-Blazos A, Wattanapenpalboon $\mathrm{N}$, et al. (2001) Skin wrinkling: can food make a difference? $J$ Am Coll Nutr 20, 71-80.

11. Boelsma E, Van de Vijer LPL, Goldbohm A, et al. (2003) Human skin condition and its associations with nutrient concentrations in serum and diet. Am J Clin Nutr 77, 348-355.

12. Cosgrove MC, Franco OH, Granger SP, et al. (2007) Dietary nutrient intakes and skin-aging appearance among middleaged American women. Am J Clin Nutr 86, 1225-1231.
13. Nagata C, Nakamura K, Oba S, et al. (2009) Association of intakes of fat, dietary fiber, soy isoflavone, and alcohol with uterine fibroids in Japanese women. Br J Nutr 101, 1427-1431.

14. Sasaki S, Kobayashi M \& Tsugane S (1999) Development of substituted fatty acid composition table for the use in nutritional epidemiologic studies for Japanese populations: its methodological backgrounds and the evaluation. $J$ Epidemiol 9, 190-207.

15. Shimizu H, Ohwaki A, Kurisu Y, et al. (1999) Validity and reproducibility of a quantitative food frequency questionnaire for a cohort study in Japan. Jpn J Clin Oncol 29, 38-44.

16. Suzuki I, Kawakami N \& Shimizu H (1998) Reliability and validity of a questionnaire for physical activity in epidemiological studies. J Epidemiol 8, 152-159.

17. Shimizu H (1996) The Basic Report on Takayama Study. Gifu, Japan: Department of Public Health, Gifu University School of Medicine.

18. Willett W (1990) Implication of total energy intake for epidemiological analyses. In Nutritional Epidemiology, pp. 245-271 [W Willett, editor]. Oxford: Oxford University Press.

19. Guinot C, Malvy DJM, Ambrosine L, et al. (2002) Relative contribution of intrinsic vs extrinsic factors to skin aging as determined by a validated skin age score. Arch Dermatol 138, $1454-1460$.

20. Cua AB, Wilhelm KP \& Maibach HI (1990) Elastic properties of human skin: relation to age, sex, and anatomical region. Arch Dermatol Res 282, 281-288.

21. Piérard GE, Henry F, Castelli D, et al. (1998) Aging and rheological properties of facial skin in women. Gerontology 44, 159-161.

22. Lansdown ABG (2004) Nutrition 2: a vital consideration in the management of skin wounds. Br J Nurs 13, 1199-1210.

23. Brincat MP (2000) Hormone replacement therapy and the skin. Maturitas 35, 107-117.

24. Dunn LB, Damesyn M, Moore AA, et al. (1997) Does estrogen prevent skin aging? Results from the First National Health and Nutrition Examination Survey (NHANES I). Arch Dermatol 133, 339-342.

25. Prentice R, Thompson D, Clifford C, et al. (1990) Dietary fat reduction and plasma estradiol concentration in healthy postmenopausal women. The Women's Health Trial Study Group. J Natl Cancer Inst 82, 129-134.

26. Kaneda N, Nagata C, Kabuto M, et al. (1997) Fat and fiber intakes in relation to serum estrogen concentration in premenopausal Japanese women. Nutr Cancer 27, 279-283.

27. Eberlein-König B, Placzek M \& Przybilla B (1998) Protective effect against sunburn of combined systemic ascorbic acid (vitamin C) and D- $\alpha$-tocopherol (vitamin E). J Am Acad Dertmatol 38, 45-48.

28. Morganti P, Fabrizi G \& Bruno C (2004) Protective effects of oral antioxidants on skin and eye function. Skinmed 3, 310-316.

29. Heinrich U, Tronnier H, Stahl W, et al. (2006) Antioxidant supplements improve parameters related to skin structure in humans. Skin Pharmacol Physiol 19, 224-231.

30. Berardesca E (1997) EENCO guidance for the assessment of stratum corneum hydration: electrical methods. Skin Res Technol 3, 126-132. 